\title{
CARACTERIZACIÓN DE UNA NOCIÓN DE SiMUlaCión A PARTIR DE PRÁCTICAS EXPERIMENTALES
}

\author{
PÍO GARCÍA
}

\begin{abstract}
Computer simulations have been characterized by making a sharp contrast with others scientific practices. In this paper we propose to understand computer simulations from interventive practices in a particular field: life sciences. We defend two theses. First, to characterize computer simulations requires to understand experimental practices. Second, we argue that to understand experiments requires a previous notion of simulation. In other words, we defend that the practices associated with simulations and experimentation are two complementary aspects, at least in life sciences.
\end{abstract}

Keywords: Computer simulation; in vivo - in vitro dichotomy; experimental practices.

\section{Introducción}

Las técnicas de simulación han ido ganando terreno en diferentes disciplinas de la actividad científica contemporánea. La aparente novedad de esta metodología requiere de una elucidación de su identidad que permita, entre otras cosas, una evaluación de su alcance epistémico. Así como las técnicas experimentales constituyeron un desafío a la dicotomía tradicional entre teoría y observación, probablemente las simulaciones computacionales tengan algo para aportar al contexto de las estrategias científicas. De esta manera, en los últimos años se ha intentado, de diferentes formas, una caracterización filosóficamente relevante de las simulaciones computacionales. A partir del trabajo pionero de Rohrlich y Humphreys en los años 90 del siglo pasado (Rohrlich 1990; Humphreys 1990), la elucidación de lo que son las simulaciones computacionales se constituyó como problema central. Así, las simulaciones computacionales se entienden como un tipo particular de proceso (Hartmann 1996), a partir de una estructura que no se reduce a los modelos conocidos - las llamadas plantillas computacionales - (Humphreys 2004) o desde los "trucos" computacionales que permiten implementar modelos científicos (Winsberg 1999; Winsberg 2003).

Otra vía para abordar el problema de las simulaciones ha destacado el contraste entre éstas técnicas y diversas prácticas científicas. De esta manera habría una diferencia nítida entre prácticas representacionales vinculadas con la modelización, entre las cuales estarían las simulaciones computacionales, y prácticas interventivas vinculadas con sistemas físicos. Si las simulaciones computacionales ocupan un espacio genuino en la actividad científica, entonces estarían más fuertemente emparentadas con la teorización y en un claro contraste con la experimentación.

Principia 19(2): 217-234 (2015).

Published by NEL — Epistemology and Logic Research Group, Federal University of Santa Catarina (UFSC), Brazil. 
Sin embargo, los intentos de obtener una caracterización de lo que son las simulaciones computacionales han tenido que enfrentarse al desafío de si hay algo específico en esta práctica científica. Para algunos son sólo odres nuevos para vinos viejos (Frigg \& Reiss 2009). Además aquellos que llevan adelante esta empresa han tenido que dar cuenta de la diversidad de prácticas científicas asociadas con las simulaciones. Así, en los últimos años se han publicado numerosos estudios de caso sobre simulaciones computacionales. A pesar de las excelentes descripciones de prácticas puntuales, no parece, en principio, que este tipo de tarea, fuertemente descriptiva, pueda aportar a una caracterización de lo que son las simulaciones computacionales. Esta tensión entre generalidad y capacidad descriptiva no es exclusiva de este campo, pero es particularmente crítica en el ámbito de las simulaciones dada su ubicuidad y diversidad. En este contexto no parece sencillo dar con una caracterización que sea pertinente y que, a la vez, de cuenta de las prácticas en ciencia.

En el presente trabajo propondremos una manera de abordar la cuestión de la caracterización de las simulaciones computacionales adoptando un punto de vista alternativo. En cierto sentido nuestra propuesta asocia ambas empresas: aquella que intenta llevar adelante una elucidación conceptual y aquella que pretende aprender de las prácticas científicas. Para esto, analizaremos un contexto científico específico, vinculado con las ciencias de la vida, con el objetivo de comprender lo que son las simulaciones computacionales. En particular defenderemos dos tesis. En primer lugar que, para elucidar lo que son las simulaciones computacionales, se requiere de una comprensión de las prácticas experimentales. En segundo lugar argumentaremos que para comprender lo que son los experimentos se requiere de una noción de simulación. En otras palabras, defenderemos que las prácticas asociadas con la simulación y con la experimentación constituyen dos aspectos complementarios en ciencias de la vida como la bioquímica o la fisiología.

Nuestra propuesta involucra replantear la tarea de caracterización de las simulaciones computacionales. Dicha tarea parece más fructífera si, en lugar de proponer un contraste de tipo ontológico entre aspectos abstractos y representacionales y aspectos concretos e interventivos, se destacan las prácticas simulativas en los experimentos. Como dijimos más arriba: para entender lo que son las simulaciones en las ciencias de la vida, entre ellas las simulaciones computacionales, se necesita tomar en consideración, de una forma específica, el contexto experimental. Asimismo para poder caracterizar lo que son los experimentos, en estas disciplinas, se requiere comprender el rol que desempeñan las simulaciones. En este esquema, como veremos más adelante, son centrales la relación entre condiciones in vivo e in vitro.

Sin embargo, la relación que estamos proponiendo entre prácticas experimentales y simulaciones parece ir en contra de nuestras intuiciones. ${ }^{1}$ En principio, la historia de la experimentación y de las simulaciones computacionales sugieren un orden entre estas estrategias científicas que contradice nuestra propuesta. De acuerdo con esta

Principia 19(2): 217-234 (2015). 
objeción, las técnicas experimentales han sido parte de disciplinas como la bioquímica o la fisiología desde sus inicios. En cambio las simulaciones sólo han comenzado a utilizarse hace relativamente poco tiempo. Pero, tal como argumentaremos en este trabajo, las prácticas metodológicas simulativas han sido muy importantes en ciencias de la vida antes del advenimiento de las simulaciones computacionales. Y esta noción de simulación juega un papel central no sólo en prácticas experimentales sino en las simulaciones computacionales.

Organizaremos nuestro trabajo de la siguiente manera. En la segunda sección realizaremos una revisión de la literatura reciente sobre filosofía de las simulaciones computacionales. Destacaremos en esta sección la relevancia que ha tenido en la literatura las consideraciones ontológicas en la tarea de contrastar simulaciones con experimentos. En la tercer sección haremos una presentación esquemática y breve de las simulaciones computacionales habitualmente utilizadas en bioquímica. En la cuarta sección sugeriremos que, a partir de la relación in vivo - in vitro, es posible comprender el vínculo entre prácticas experimentales y simulaciones. En la sección final de este trabajo realizaremos una recapitulación de las tesis centrales defendidas.

\section{Acerca de la noción de simulación computacional}

Como planteábamos en la introducción, un camino habitual para aproximarse a las simulaciones computacionales ha sido su comparación con los experimentos. De esta manera se han destacado aspectos ontológicos, inferenciales, metodológicos y funcionales, siendo la preocupación filosófica central la cuestión de la validez de las simulaciones o, en términos más generales, sus credenciales epistémicas.

Esta perspectiva comparativa reaparece de manera insistente en publicaciones recientes, posiblemente por lo que sugiere la expresión "experimento numérico" referido a las simulaciones - pero también como una manera de poner en cuestión el lugar de este tipo de práctica, relativamente nueva, en el ámbito de categorías tradicionales de la filosofía de la ciencia. En esta línea se ha dicho que las simulaciones "están en algún lugar intermedio entre la ciencia (física) teórica tradicional y sus métodos empíricos de observación y experimentación” (Rohrlich 1990).

La discusión acerca de los aspectos ontológicos y epistémicos asume el esquema típico de sistema estudiado y "sistema referencia" (target system). ${ }^{2}$ La cuestión de la validez se entiende en términos de la relación entre un sistema (experimento o simulación) y el "sistema referencia". Francesco Guala ha sugerido, por ejemplo, que la diferencia entre un experimento y una simulación estaría en el tipo de justificación de las inferencias que relacionan el sistema estudiado con el "sistema referencia" (2002). En el caso de un experimento, si en el sistema estudiado y en el "sistema referencia" están operando las mismas causas, entonces las inferencias que vincu-

Principia 19(2): 217-234 (2015). 
lan ambos sistemas estarían "fuertemente justificadas". ${ }^{3}$ Por el contrario, entre una simulación computacional y un sistema simulado ("sistema referencia") sólo habría una correspondencia abstracta y formal. Gilbert y Troitzsch (2005) también parecen suponer que las diferencias entre una simulación y un experimento estarían representadas por el tipo de relación que dichos sistemas establecen con sus "sistemas referenciados". En el caso de un experimento aquello que se manipula es un "objeto real", mientras que en una simulación aquello que se estudia o manipula es un modelo (Gilbert \& Troitzsch 2005, p.14). De manera implícita se está estableciendo una caracterización en términos de la cercanía ontológica con el fenómeno estudiado.

Este punto de vista ontológico también está presente en propuestas como la de Wendy Parker. En Parker (2009) se sugiere que la relación que debería considerarse entre un sistema y su "sistema referencia" es el de "similaridad relevante". Un experimento es caracterizado como "una actividad de investigación en la cual se interviene en un sistema para ver cómo las propiedades que nos interesan cambian a partir de dicha intervención" (Parker 2009, p.487). Parece que, en principio, lo que distingue a una simulación de un experimento es la intervención. Y, en esta perspectiva, sólo en un sistema "material" se pueden realizar actividades interventivas. Este punto de vista aparece más claro cuando Parker presenta la idea de un "estudio de simulación computacional". Cuando consideramos a un sistema computacional como un sistema físico - un programa computacional implementado en una máquina física -, entonces somos capaces de identificar actividades interventivas genuinas.

Esta perspectiva centrada en la "materialidad" de los experimentos ha sido matizada de diferentes maneras. Morgan (2005) argumenta en favor de este punto de vista pero enfatizando los aspectos inferenciales involucrados. Parece indudable que los experimentos y las simulaciones tienen "poderes epistémicos" diferentes. Y esta diferencia se podría explicar, nuevamente, porque, en el caso de los experimentos, el sistema manipulado y el "sistema referencia" están hechos de la "misma cosa" (same stuff). Además, las simulaciones, dependiendo por completo de sus modelos, no pueden "sorprender" de manera genuina a los científicos. La diferencia ontológica entre simulaciones y experimentos genera una diferencia epistémica. Hay un sentido en el cual las simulaciones pueden ser experimentos, pero de una clase particular: "no materiales". Por tanto, a pesar del cambio terminológico, no habría ninguna modificación en un sentido ontológico - y por ende tampoco habría una modificación en la consideración del alcance epistémico de las prácticas simulativas y experimentales. ${ }^{4}$

En contraste con estas perspectivas, hay algunos filósofos que han destacado aspectos metodológicos o funcionales en la relación entre experimentos y simulaciones. En este sentido, Eric Winsberg sugiere que la distinción entre simulaciones y experimentos se debería realizar en términos del tipo de inferencia que surge del conocimiento básico supuesto (background knowledge). En el caso de las simulaciones, este conocimiento básico es el que nos permite construir modelos matemáticos y

Principia 19(2): 217-234 (2015). 
computacionales. Y la confiabilidad del modelo descansa en la confiabilidad del conocimiento básico supuesto. Asimismo, Margaret Morrison ha destacado la dimensión funcional en la relación entre simulaciones y experimentos. En (Morrison 2009) se defiende que, por la manera en la cual se construyen los modelos, habría semejanzas funcionales entre simulaciones e instrumentos de medida. En este sentido, se podría hablar de simulaciones que funcionan como instrumentos experimentales de medición.

También se presenta la relación entre simulación y experimento en términos de sustitución. Cuando no es posible hacer un experimento, por razones prácticas o éticas, entonces como una alternativa se implementa una simulación. Así, en algunos campos científicos una simulación puede cumplir las funciones de y, en este sentido, ser considerada como si fuese un experimento porque permite investigar un fenómeno no accesible a la experimentación. Una distinción que se discute en este contexto es entre los conceptos de representación y ser representativo. De esta manera se pretende dar cuenta de dos maneras en las cuales un sistema puede suplir a o estar en lugar de (stand in for) otro. Una relación de representación parece típicamente descansar en una vinculación formal, mientras que una relación representativa sería una forma de suplir que es similar a la forma en la cual "una parte puede estar por el todo". Por esta razón se habla de "ser parte de lo mismo", donde lo mismo parece entenderse como la misma clase de cosa o materia (being of the same stuff) (Peschard 2013). En términos más puntuales se podría reseñar las relaciones entre experimentos y simulaciones computacionales de la siguiente manera. En primer lugar, como ya hemos visto, se estima que la forma de entender el problema es analizando tanto las simulaciones como los experimentos en relación con el "sistema referencia" (target). En segundo lugar hay un conjunto de "intuiciones básicas" que funcionan como punto de partida y guías para la indagación. Siguiendo a Peschard, se podría decir que en un experimento, se interactúa de alguna forma con el sistema bajo estudio y se le "da la oportunidad (al sistema) de expresarse por medio de los efectos causales de los instrumentos" (Peschard 2013). Por contraste, si en una simulación se puede hablar de algún tipo de "expresión", sería únicamente la del modelo utilizado. De aquí que "la distinción epistémica sea clara: la experimentación produce información acerca de cómo se comporta el sistema, mientras que la simulación produce información acerca del modelo" (Peschard 2013) . La conclusión es muy similar a la ya citada de Morgan. Además, la discusión acerca de la sustitución vuelve sobre las distinciones ontológicas como una forma de justificar las diferencias de "poder epistémico" entre simulaciones y experimentos.

Si bien en la literatura filosófica que compara experimentos con simulaciones se destacan aspectos inferenciales, metodológicos y funcionales, son los aspectos ontológicos los que juegan un papel central para evaluar el valor epistémico de estas prácticas. Además, la oposición entre simulaciones y experimentos parece centrarse 
en la relación entre modelos y sistema físico sobre el que se interviene. En el presente trabajo destacaremos mas bien los aspectos metodológicos de la relación entre simulaciones y experimentos, a la vez que presentaremos la relación entre modelos y "sistema físico" de una manera diferente. Este cambio de perspectiva permite dar cuenta de la función epistémica de las simulaciones y experimentos. Veamos primero qué se entiende por simulación computacional en ciencias de la vida, para luego presentar los contextos científicos a partir de los cuales elucidar una noción de simulación experimental.

\section{Simulaciones computacionales en ciencias de la vida}

Las simulaciones computacionales más utilizadas en ciencias naturales son aquellas basadas en ecuaciones. ${ }^{5}$ Una de las razones por las cuales las simulaciones computacionales son importantes en ciencias naturales es porque esta herramienta permite "explorar" un espacio de soluciones:

[...] muchos fenómenos naturales son no lineales y una cambio pequeño en una variable puede producir un cambio enorme en otra. Puesto que relativamente pocos problemas no lineales pueden ser resueltos por métodos analíticos, la computadora nos da una nueva herramienta para explorar fenómenos no lineales. Otra razón de la importancia de la computadora es el creciente interés en sistemas complejos con muchas variables o con muchos grados de libertad. (Gould, Tobochnik \& Christian 2007, p.2)

Las simulaciones computacionales basadas en modelos o en ecuaciones suelen ser descriptas como "un programa que se ejecuta en una computadora y que usa métodos paso a paso para explorar la "conducta" aproximada del modelo matemático" (Winsberg 2014). ${ }^{6}$

En bioquímica, las simulaciones suelen ser utilizadas para estudiar e investigar aspectos dinámicos de un sistema dado, como los involucrados en una reacción metabólica, típicamente a través de un modelo matemático implementado en un sistema digital. La tarea habitual de estas simulaciones suele ser resolver ecuaciones diferenciales ordinarias (EDO) que caracterizan a un sistema mediante métodos aproximativos. Incluso cuando la dinámica temporal de una reacción bioquímica se pueden expresar por medio de EDO, las ecuaciones suelen ser no lineales y por ende se requiere de una simulación (Mendes 1994). Es importante destacar que los modelos computacionales no son los modelos matemáticos originales sino una implementación de los mismos. Probablemente el aspecto más saliente de este proceso de implementación sea la discretización de los modelos originales. El desideratum de los modelos computacionales es la 'tratabilidad'. Para lograr este objetivo no sólo se recurre a la ya mencionada discretización sino también a diversos recursos meramente

Principia 19(2): 217-234 (2015). 
computacionales que permitan llegar a alguna solución aproximada (Cfr. Humphreys 2004, cap. 3).

Sin embargo, para aquellos que trabajan con simulaciones éstas son algo más que meros "trituradores de números". Cuando se usan simulaciones "habitualmente el objetivo principal es la comprensión (insight) más que simplemente números" (Gould, Tobochnik \& Christian 2007, p.3). Así, por ejemplo, en bioquímica se suelen señalar los siguientes roles para las simulaciones de cinética metabólica:

[...] Las simulaciones sirven para poner a prueba la consistencia de nuestras teorías a través de un comportamiento observado; nos permiten hacer preguntas como-si que revelan propiedades no intuitivas del metabolismo; pueden ser usadas para estimar (find estimatives) parámetros cinéticos y para el análisis genético funcional; también pueden ser utilizadas como una herramienta educativa. (Mendes 1997, p.61)

Las simulaciones permitirían poner a prueba hipótesis, realizar indagaciones contrafácticas y estimar valores de parámetros, entre otras funciones. Sin embargo, estos usos o funciones de las simulaciones están supeditados al valor mimético de esta herramienta. En tanto las simulaciones computacionales permiten estar en lugar del sistema estudiado o "imitando" aspectos relevantes del mismo, se pueden utilizar luego para poner a prueba hipótesis o realizar investigaciones confiables de estados alternativos. En gran medida esta capacidad mimética está asociada con la capacidad representacional de los modelos utilizados por una simulación, pero también parece estar vinculada con las computaciones (aproximaciones) que permiten dichos modelos y, en términos más generales, con el despliegue de escenarios dinámicos. En la sección siguiente volveremos sobre esta cuestión.

Las primeras simulaciones computacionales en bioquímica utilizaron una computadora analógica mecánica para resolver las EDO. Más tarde, se utilizaron otros tipos de equipos electrónicos analógicos (Mendes 1994). Así por ejemplo, Britton Chance, en la primera mitad del siglo pasado, es uno de los primeros en utilizar como un simulador este tipo de recursos analógicos. En Chance (1943), este bioquímico encuentra por primera vez una evidencia fuerte en favor de la teoría de Michaelis y Menten acerca de la velocidad de diversas reacciones enzimáticas. Dicha velocidad suele estar vinculada con alguna enzima o sustrato que suele acelerar la reacción (función catalizadora). Para poner a prueba la teoría de Michaelis y Menten, Chance utiliza un aparato con dos jeringas que le permite mezclar compuestos de manera controlada. Con este aparato Chance se puede focalizar en la influencia que tiene en la velocidad de reacción las enzimas, sustratos y compuestos intermedios. Pero,

[...] bajo las condiciones experimentales se encontró que los estados estacionarios existen sólo por una fracción de segundos. Para poder determinar si las porciones de las curvas [de los datos que no pueden examinarse expe-

Principia 19(2): 217-234 (2015). 
rimentalmente] satisfacen la teoría de Michaelis, se requieren soluciones de las ecuaciones diferenciales. (Chance 1943, p.574)

Esta tarea encontrar las soluciones que no aparecen en los registros experimentales se realizó con un "analizador diferencial", una computadora analógica para integrar las EDO. ${ }^{7}$ Esto es: para dar cuenta de la tarea mimética, en este caso, habría que tomar en cuenta tanto el instrumento experimental - el mezclador de compuestos - como el "analizador diferencial". En el artículo citado de Chance parece claro que sólo con ambos tipos de simulación - experimental y computacional - se pudo poner a prueba la teoría de Michaelis y Menten.

Desde la década del 60 del siglo pasado, casi todas las simulaciones se llevan a cabo a través de computadoras digitales. Las simulaciones computacionales son ampliamente utilizadas para estudiar sistemas como las reacciones metabólicas. En este grupo, existen programas como Metamod y GEPASI ${ }^{8}$ o Scamp que intentan simular la dinámica de las reacciones bioquímicas. La tarea aquí es también la aplicación de métodos aproximativos para resolver las EDO que describen a un sistema. Este tipo de programa computacional puede ser considerado como un asistente en la tarea de construcción de modelos, ya que "traducen" fórmulas bioquímicas en ecuaciones matemáticas. Así, por ejemplo, el usuario tiene que suministrarle al sistema información sobre la estructura del mecanismo considerado, la cinética de las reacciones y la concentración inicial de especies químicas; entonces el sistema computacional "construye" las ecuaciones diferenciales que caracterizan a dicho sistema. Programas como el GEPASI también se utilizan generalmente para el estudio de las propiedades del estado estacionario de un mecanismo químico. En particular, este programa ha sido utilizado para estudiar interacciones de enzimas en rutas bioquímicas. ${ }^{9}$

Además de esta función de "traducción", estos programas son utilizados para poner a prueba hipótesis. Se pueden, de esta manera, construir escenarios en donde desarrollar las implicaciones de supuestos particulares. Por ejemplo, a través de un módulo del GEPASI que sirve para explorar el espacio de parámetros (scanning parameter space), se pueden encontrar que hay señales metabólicas que, en determinados rangos de concentraciones de enzimas, funcionan como un "interruptor". La exploración de parámetros permite estimar en qué rango de valores se puede encontrar este "interruptor" (Cfr. Mendes 1997). Otra tarea en la cuál el sistema puede colaborar es en la identificación de los parámetros del modelo. Dicha identificación permite, entre otros objetivos, estimar las velocidades de reacción que no pueden ser examinadas experimentalmente (Riznichenko et. al. 2009).

Pero probablemente uno de los usos más interesantes de las simulaciones computacionales está vinculado con las condiciones in vivo - in vitro. ${ }^{10}$ En principio parece que las condiciones in vivo están casi por definición vinculadas con el contexto experimental. Y si bien esto es cierto en la mayoría de los casos, dada la complejidad de las 
condiciones in vivo, en algunas ocasiones se requiere apelar a otros recursos más allá de los estrictamente experimentales. Esto puede verse en dos situaciones. La primer situación es más general y está vinculada con la complejidad de las condiciones in vivo tales como las que se dan en la célula. Dicha complejidad involucra, por ejemplo, interacciones no lineales entre moléculas. La modelización, en consecuencia, debe dar cuenta de las características del fenómeno considerado. Por tanto

Sólo el análisis matemático cuantitativo, mucha veces más allá de lo que es posible analíticamente, es un prerequisito para comprender la célula viva. (Snoep, Mendes \& Westerhoff 1999, p.25)

Las simulaciones computacionales son las que permiten este análisis cuantitativo y, por ende, simular las condiciones in vivo. Las aproximaciones a fenómenos no lineales de las simulaciones computacionales son, de acuerdo con esta perspectiva, un recurso indispensable para "comprender" la célula viva.

La segunda situación en donde las condiciones in vivo parecen ir más allá de las condiciones experimentales esta vinculado con el área ya citada de las reacciones metabólicas. Cuando lo que se busca es determinar al responsable de una tasa de reacción parece que la respuesta está en la identificación de un agente particular, esto es una enzima o algún tipo de catalizador. En términos más generales, la búsqueda se centra en "propiedades locales" de las enzimas y de los procesos cinéticos. Sin embargo, en muchas ocasiones, las condiciones in vivo indican que el control de la reacción se debe considerar como "global". De esta manera, para lograr una modelización más apropiada de las condiciones in vivo se requiere de dar cuenta de este aspecto "global" del control de las reacciones. Para situaciones de este tipo, más comunes de lo que se cree, hay una teoría denominada Control de Análisis Metabólico (MCA por sus siglas en inglés). MCA es un tipo de análisis que ya es considerado estándar para abordar la cinética de una reacción. ${ }^{11}$ Una de las contribuciones más importentes del MCA es que el control de la reacción es considerada como una propiedad del sistema total más que una propiedad de las enzimas particulares (Snoep, Mendes \& Westerhoff 1999, p.26). Sistemas como el GEPASI permiten modelizar a través de los supuestos del MCA.

A través de estos dos ejemplos se puede ver que las condiciones in vivo no necesariamente están conectadas con los contextos experimentales tradicionales. Curiosamente, este es un primer paso importante para defender nuestra propuesta de que, para caracterizar a las simulaciones computacionales, se necesita tomar en consideración las condiciones experimentales de una forma específica. El punto es que la relación entre las condiciones in vivo e in vitro son estas condiciones específicas a las que hacíamos referencia. El vínculo entre simulaciones computacionales y prácticas experimentales lo constituyen las condiciones in vivo e in vitro. Y habitualmente se supone que estas condiciones son sólo parte del contexto experimental. Por esta ra-

Principia 19(2): 217-234 (2015). 
zón, para que las condiciones in vivo e in vitro puedan cumplir el rol de vínculo que estimamos tiene, teníamos que insinuar que las simulaciones computacionales en ocasiones son el único medio del que se dispone, dada su complejidad, para acceder a sistemas vivos.

Antes de desarrollar las implicaciones de esta relación para nuestra tarea de caracterizar a las simulaciones computacionales veamos más en detalle qué implican las condiciones in vivo e in vitro en el ámbito experimental de las ciencias de la vida.

\section{Contextos experimentales y simulaciones: in vivo - in vitro}

En el campo de las ciencias de la vida las expresiones in vivo - in vitro son habituales para hacer referencia a contextos experimentales diversos. ${ }^{12}$ En la presente sección argumentaremos que hay condiciones experimentales - las involucradas en la relación in vivo - in vitro que pueden ser consideradas simulaciones genuinas. Esta generalización de la noción de simulación, combinado con el resultado obtenido en la sección anterior, nos permitirá defender el vínculo estrecho que hay entre prácticas experimentales y simulaciones computacionales. Así, en esta sección sugeriremos que la noción de simulación no debería circunscribirse al ámbito computacional. Para esta tarea abordaremos aquellos aspectos metodológicos que caracterizan a la relación in vivo - in vitro.

Las situaciones y contextos en donde son utilizadas las condiciones in vivo son de naturaleza muy variada: incluye estudios en tejidos, en órganos aislados (llamados a veces ex vivo) e incluso en los llamados "modelos animales". Por contraste se realizan estudios in vitro cuando se requiere un mayor control sobre condiciones experimentales específicas. Desde los orígenes mismos de la bioquímica y la fisiología, fue metodológicamente importante la contraposición entre condiciones in vivo e in vitro (Holmes 1991).

Veamos primero algunos aspectos conceptuales de los estudios in vitro. Roger Strand, caracteriza, en un artículo acerca de la confiabilidad de los experimentos en bioquímica, lo que él llama "estudios de efectos in vitro" (Strand, Fjelland \& Flatmark 1996). La denominación de Strand está vinculada con la intención de este científico de remarcar que se asume en este tipo de estudio que el fenómeno de interés es

[...] un cambio o diferencia en un aspecto biológico o parámetro a partir de una perturbación del sistema in vitro o del sistema in vitro comparado con un sistema de control que no esté sujeto a la perturbación [del diseño experimental] (Strand, Fjelland \& Flatmark 1996, p.2)

El objetivo general de esta clase de estudio es obtener conocimiento de un fenómeno biológico en un organismo o sistemas de organismos (de un sistema in vivo). 
Además, se destaca que esta clase de estudio se realiza porque resulta metodológicamente imposible, no conveniente o éticamente cuestionable estudiar el fenómeno de interés de manera directa (el sistema in vivo). Por esta razón un sistema experimental sustituto - in vitro - se construye a partir de una o más partes de un sistema in vivo. De esta manera, información acerca del sistema in vivo se infiere interpretando la evidencia experimental de un sistema sustituto análogo (putative analog), esto es de un fenómeno o sistema in vitro. El sistema in vitro tiene que ser diferente de aquél que se quiere estudiar para evitar o paliar las dificultades, principalmente metodológicas o éticas en algunos casos. Suele ocurrir que el diseño de un sistema in vitro involucra un tipo de "ruptura en un sentido físico" (physical disruption) (Strand, Fjelland \& Flatmark 1996) del sistema in vivo e involucra a su vez un subsiguiente proceso que permita aislar una o más de sus partes con la intención de lograr acceso experimental a una característica o parámetro, o para reducir la complejidad de interacciones con el fenómeno interferido. A los fines de lograr una interpretación adecuada, los investigadores, continúa Strand, suponen por lo menos que las condiciones seleccionadas son relevantes y que "imitan" de manera no trivial el sistema in vivo. Es importante resaltar que este sentido de simulación ha estado presente en el campo de las ciencias de la vida mucho antes del advenimiento de las computadoras. Podría verse esta relación en términos de una distinción entre condiciones naturales y artificiales. Así, habría una línea divisoria clara y fija entre la simulación y aquello que es simulado. Esto es: entre lo natural y lo artificial. Sin embargo, una perspectiva metodológica y móvil parece más adecuada para caracterizar la relación in vivo - in vitro $\mathrm{y}$, por ende, la relación de simulación.

A los fines de hacer más clara la discusión tomemos como ejemplo las investigaciones vinculadas con el desarrollo de productos farmacéuticos. Entre las dificultades más importantes en este campo está la toxicidad no prevista y las fallas en reproducir el mecanismo que debería modificar la afección del modo esperado. Este fracaso puede deberse, entre otras razones, a una "inapropiada absorción, distribución, metabolismo o excreción” (llamadas "propiedades” ADME) (Williams 2011). Así, se realizan experimentos in vitro porque

La disolución de drogas es casi imposible (nearly impossible) de estudiar en el medio que se supone que debe ocurrir, esto es, el tracto gastrointestinal humano. Por esta razón como un sustituto, se requiere la puesta a prueba de la disolución in vitro para asegurarse que, en cada partida de la droga formulada, esta se disuelve a una tasa constante. (Johnson 2007, p.2)

Esta descripción se corresponde con el contexto experimental, pero podría ser utilizada, casi sin modificaciones, para caracterizar una simulación - entendida como un sustituto o estar en lugar de - Esta relación de simulación tiene un aspecto imitativo y uno "disruptivo". Estos aspectos se evidencian cuando se pone el foco en los 
aspectos metodológicos. En la colección de artículos recién citada lo que se pretende mostrar es cómo modelar el proceso de una propiedad particular (la disolución) en el contexto apropiado, con el objetivo de obtener indicios acerca de los factores que pueden ser relevantes para controlar la tasa de disolución. Dicha modelización permitiría, entre otras cosas, predecir una correlación entre la disolución in vitro y el perfil de tiempo in vivo de la droga en la sangre. La correlación entre condiciones in vitro y condiciones in vivo en el caso del desarrollo de drogas es definida como

[...] un modelo (matemático) que describe la relación entre una propiedad de un dosaje (usualmente la propiedad de interés es tasa de disolución) y una respuesta in vivo relevante (v.gr. Cantidad de droga absorbida o concentración plasmática de la droga). (FDA 1997, p.6)

La FDA define cinco niveles de correlación entre estas condiciones dependiendo de la combinación de propiedades ADME involucradas. En este ejemplo la relación entre condiciones in vivo e in vitro es caracterizada por medio de un "modelo de correlación" que permite estimar la confiabilidad de la dicha relación.

Ahora bien, como decíamos arriba, tanto por el esquema utilizado como por las funciones señaladas por Strand para describir la relación in vitro- in vivo, parece que es posible ver dicha relación en términos experimentales, pero también como una simulación. Y esta adscripción estaría de acuerdo con un uso terminológico extendido en el campo de la bioquímica. Así, en algunas ocasiones se habla de 'simulación' cuando se quiere hacer referencia al tipo de relación entre condiciones in vitro - in vivo (Wilke et. al. 1994). ${ }^{13}$ Es evidente que este sentido más general de simulación también serviría para comprender las simulaciones computacionales en ciencias de la vida. Este punto de vista alternativo en el cual el concepto mismo de "simulación" - en un sentido general - sirve para comprender a la noción de simulación computacional, ha sido considerada por algunos filósofos.

En esta perspectiva una simulación consiste en cualquier sistema que se cree o espera que tenga una dinámica suficientemente similar a otro sistema tal que el primero puede ser estudiado para aprender acerca del segundo. (Winsberg 2014)

También en este lugar podría ubicarse la sugerencia de Hartmann cuando presenta a las simulaciones como un proceso que tiene una relación de imitación con otro proceso. Aquí un proceso es sólo "una secuencia temporal de estados de un sistema" (Hartmann 1996).

Este punto de vista general puede ser desarrollado a partir del contexto científico particular que estamos considerando. Un estudio de simulación puede ser caracterizado como aquellos recursos metodológicos que permiten aprender de la dinámica de un sistema a partir de su comparación con la dinámica de otro sistema. Es más, el 
alcance y confiabilidad de la relación mimética depende también de los mencionados recursos metodológicos.

Si un estudio de simulación se caracteriza por sus aspectos miméticos, un experimento podría ser caracterizado, siguiendo posiciones ya clásicas en filosofía de la ciencia, por sus aspectos interventivos. Sin embargo, habría experimentos en donde los aspectos simulativos tienen un papel central. Tal es caso de algunos contextos experimentales en ciencias de la vida. Los estudios in vitro califican como experimentos en el sentido de realizarse en sistemas físicos y de involucrar aspectos interventivos. Pero también pueden ser considerados como simulaciones en tanto, estando en lugar de otro sistema, cumplen la función de permitir el estudio de dicho sistema imitando su dinámica. De esta manera podríamos tener además de las simulaciones computacionales, las simulaciones experimentales ${ }^{14}$ (sistemas in vitro). Ambos tipos de simulación (la computacional y la in vitro) funcionan como un sustituto controlable de un sistema in vivo y suelen tener como finalidad estudiar aspectos dinámicos de dichos sistemas biológicos (sistemas in vivo).

Ahora bien, como dijimos más arriba, el que un sistema in vitro sea una simulación parece depender de la manera en la cual cumple la función de permitir estudiar un sistema imitando la dinámica de otro que sea más accesible o controlable. Pero, como parece seguirse de esta manera de presentar el problema, esta distinción entre simulación (estudio in vitro) y aquello que es simulado (sistema in vivo) no es fija. En particular la adopción de una perspectiva metodológica e histórica permite presentar más claramente este aspecto. En un artículo reciente, Winsberg nos invita a imaginar dos científicos que pretenden investigar aspectos del comportamiento de fluidos, pero mientras uno de ellos utiliza un tanque de agua, el otro utiliza una computadora (Winsberg 2009). En principio las diferencias entre ambas estrategias podrían resumirse de la siguiente manera: mientras en el primer caso un científico "genera conocimiento empírico nuevo acerca de los fluidos a través de la manipulación de un fluido real", el otro científico "sólo explora las consecuencias" del conocimiento existente (representado por el modelo). Así, habría una diferencia significativa entre manipular un objeto (real) de interés y manipular - si hay algo como esto - un modelo de dicho objeto.

Sin embargo, como lo destaca muy bien Winsberg, es habitual que en los experimentos no se manipule el objeto de interés sino otro que puede ser "relevante". En el caso del científico que manipula un tanque de agua lo hace para comprender el "objeto de interés" o el sistema que se quiere estudiar ("sistema referenciado"). En este sentido, podemos considerar a las condiciones in vitro como una simulación experimental - de las condiciones in vivo. Pero podríamos avanzar en esta dirección aún más.

Hasta aquí se está suponiendo que la modelización del fenómeno a estudiar se corresponde con el sistema computacional o el sistema in vitro. Sin embargo, este 
no es siempre el caso. En diversas áreas es muy común el uso de modelos denominados in vivo. Podemos encontrar varios ejemplos en los manuales de protocolos de investigación sobre nuevas drogas. En un artículo reciente al preguntarse por qué se necesitan "modelos in vivo" de procesos tumorales, se indica que no es evidente cuál podría ser el contexto relevante para determinar el alcance de una droga determinada (Eccles 2001). En general estos modelos in vivo suelen hacer referencia a modelos animales. Con "modelo animal" se quiere señalar no sólo que se usan animales para experimentar sino que se encuentran aquí distintos modos y niveles de intervención: desde intervención en el proceso que se quiere estudiar hasta la modificación genética de los especímenes para hacer el modelo más "semejante" al sistema a estudiar, tales como los ratones NSG (llamados también "modelos de ratones humanizados").

Decíamos más arriba que este aspecto "móvil" de la relación de simulación puede acentuarse destacando los aspectos metodológicos desde una perspectiva temporal. A medida que se va ganando comprensión y control sobre mecanismos biológicos es posible, al menos en algunos casos, sustituir estudios que se realizaban con modelos animales por estudios más acotados en tejidos. Así no parece del todo apropiado el análisis que citábamos más arriba de Strand, quien supone que con la expresión in vitro que hay una "condición artificial" en comparación con el sistema que se quiere estudiar. Siguiendo el esquema que presentábamos antes, el sistema a estudiar son justamente las condiciones in vivo. Y este suele ser el caso en algunas circunstancias. Pero típicamente la situación es otra. Lo que suele ocurrir es que ambas condiciones, in vitro - in vivo, son consideradas como artificiales, en relación con aquello que se quiere estudiar. Entonces, más que acentuar el carácter intrínseco de las nociones de in vitro o in vivo lo que parece relevante es el tipo de relación que se establece con el sistema a estudiar o, podríamos decir, con la forma en la cual se simula el sistema a estudiar. Este tipo de relación sería una forma de estar en lugar de otro sistema. Y esta forma de estar por otro se caracterizaría principalmente en términos metodológicos. Es la manera particular en la cual la simulación aísla y controla la que permite su vinculación significativa con algún aspecto del sistema que se quiere estudiar.

\section{Consideraciones finales}

Comenzamos este trabajo haciendo referencia a aquellas perspectivas que pretenden comprender las simulaciones computacionales a partir de una distinción nítida con los experimentos. Además vimos que esta distinción se realizaba, habitualmente, en términos ontológicos. En las dos últimas secciones hemos intentado dar razones por las cuales estimamos que esta forma de plantear lo que son las simulaciones computacionales es inadecuada.

A partir de lo expuesto se puede comprender mejor porqué proponíamos en la

Principia 19(2): 217-234 (2015). 
introducción entender a las simulaciones computacionales, y a los experimentos, como dos aspectos complementarios de las práctica científica en ciencias de la vida. La investigación a través de simulaciones computacionales y los experimentos, en estas disciplinas, suelen suponer un recurso metodológico central como lo son las condiciones in vivo e in vitro. Estas condiciones deben entenderse como recursos experimentales y de simulación. Por tanto, como vimos en la segunda sección, puede haber experimentos que sean considerados simulaciones. A la vez en la sección tercera defendimos que las simulaciones son una herramienta de primer orden para acceder a condiciones in vivo. Como puede verse, no intentamos aquí borrar la distinción entre experimentos y simulaciones computacionales, sino destacar que ambas prácticas deben comprenderse - y por tanto caracterizarse - tomando en consideración su interrelación. Y esta interrelación se puede establecer a través de una noción de simulación más general - y por tanto no circunscrita al ámbito computacional - y que está supuesta en las condiciones in vivo e in vitro.

No hemos arribado a una caracterización filosófica de las simulaciones computacionales como las que reseñábamos en la segunda sección del trabajo. Nuestra tarea más bien ha sido marcar la necesidad de encarar la tarea de elucidación considerando aspectos específicos de los contextos experimentales. El que un sistema simule en un sentido computacional o experimental - involucra una manera de estar en lugar de otro sistema. Y esta manera de estar en lugar de otro debería explicitarse en términos metodológicos, tal como lo hemos hecho en la sección tercera y cuarta de este trabajo. La perspectiva metodológica adoptada es central para nuestra propuesta porque así como hemos defendido que el límite entre condiciones in vivo e in vitro es móvil y dependiente del contexto también lo es el límite entre contextos experimentales y simulaciones computacionales. Este paralelismo se funda en que entre estas prácticas científicas aparentemente tan disímiles - interventivas y representacionales - están operando los supuestos metodológicos de la relación in vivo - in vitro. $\mathrm{Y}$ sin esta relación difícilmente se pueda comprender por qué simulan los programas computacionales o por qué son confiables los resultados experimentales.

La caracterización de simulación computacional — y de práctica experimental — que sugerimos aquí está fuertemente anclada en aquellas disciplinas que utilizan una noción de simulación como la supuesta en la relación entre condiciones in vivo e in vitro. Retomando el desafío que planteábamos en la introducción en relación con la tensión entre generalidad y especificidad, nuestra propuesta involucra una toma de posición fuerte. Pero, recordemos que en la introducción presentábamos este problema como una dicotomía: el estudio de casos excluía un interés genuino por elucidar qué son las simulaciones. En este trabajo hemos defendido que no necesariamente existe esta dicotomía. Probablemente el camino más adecuado sea construir caracterizaciones generales - no universales - pero significativas para luego indagar su eventual extensión a otros ámbitos.

Principia 19(2): 217-234 (2015). 


\section{Referencias}

Chance, B. 1943. The Kinetics of the Enzyme-substrate compound of Peroxidase. J. Biol. Chem. 151: 553-77.

Duran, J. 2013. A Brief Overview of the Philosophical Study of Computer Simulations. APA Newsletter 13: 38-46.

Eccles, S. A. 2001. Basic principles for the study of metastasis using animal models. In Metastasis Research Protocols Springer: 161-71.

Food/Industry, Drug Administration Guidance for, editors: Extended release oral dosage forms: development, evaluation, and application of in vitro/in vivo correlations. U.S. Department of Health and Human Services September, 1997.

Frigg, R.; Hartmann, S. 2012. Models in Science. In: E. N. Zalta (ed.) The Stanford Encyclopedia of Philosophy. Fall 2012 edition.

Frigg, R.; Reiss, J. 2009. The Philosophy of Simulation: Hot New Issues or Same Old Stew? Synthese 169(3): 593-613.

Garcia, P.; Velasco, M. 2013. Exploratory strategies: experiments and simulations. In: J. Duran (ed.) Computer Simulations and the Changing Face of Scientific Experimentation. Cambridge Scholar Publishing, p.99-108.

Garcia, P. 2006. Beyond the dichotomy in vivo - in vitro: in silico. URL: http://philsci-archive.pitt.edu/2777/i

. 2009. Discovery by serendipity: a new context for an old riddle. Foundations of Chemistry 11(1): 33-42.

- 2015. Computer simulations and experiments: in vivo in vitro conditions in biochemistry. Foundations of Chemistry 17(1): 49-65.

Gilbert, N; Troitzsch, K. G. (eds.). 2005. Simulation for the social scientist. McGraw Hill.

Gould, H.; Tobochnik, J.; Christian, W. 2007. An introduction to computer simulation methods: applications to physical systems. Pearson Addison Wesley.

Guala, F. 2002. Models, simulations, and experiments. In: Model-based reasoning, p.59-74. Springer.

Hartmann, S. 1996. The World as a Process: Simulations in the Natural and Social Sciences. In: ; R. Hegselmann et al. (eds.) Modelling and Simulation in the Social Sciences from the Philosophy of Science Point of View, p.77-100. Dordrecht: Kluwer.

Holmes, F. L. 1991. Hans Krebs. New York: Oxford University Press.

Humphreys, P. 1990. Computer simulations. Proceedings of the biennial meeting of the philosophy of science association: 497-506.

-2004. Extending ourselves: computational science, empiricism, and scientific method. New York: Oxford University Press.

Johnson, K. C. 2007. Dissolution: fundamentals of in vitro release and the biopharmaceutics classification system. Drugs And The Pharmaceutical Sciences 165.

Mendes, P. 1994. Computer simulation of the dynamics of biochemical pathways. Ph.D thesis. University of Wales: Aberystwyth.

- 1997. Biochemistry by numbers: simulation of biochemical pathways with Gepasi 3. Trends in biochemical sciences 22(9): 361-63.

Morgan, M. S. 2005. Experiments versus models: New phenomena, inference and surprise. Journal of Economic Methodology 12(2): 317-29.

Principia 19(2): 217-234 (2015). 
Morrison, M. 2009. Models, measurement and computer simulation: The changing face of experimentation. Philosophical Studies 143(1): 33-57.

Parker, W. S. 2009. Does matter really matter? Computer simulations, experiments, and materiality. Synthese 169(3): 483-96.

Peschard, I. 2013. Modeling and Experimenting. Models, Simulations, and Representations 9.

Riznichenko, G. Y. et al.. 2009. Mathematical and computer modeling of primary photosynthetic processes. Biophysics 54(1): 10-22.

Rohrlich, F. 1990. Computer Simulation in the Physical Sciences. Proceedings of the Biennial Meeting of the Philosophy of Science Association.

Snoep, J.; Mendes, P; Westerhoff, H. 1999. Teaching metabolic control analysis and kinetic modelling. The Biochemist 25: 25-8.

Strand, R.; Fjelland, R.; Flatmark, T. 1996. In vivo interpretation of in vitro effect studies with a detailed analysis of the method of in vitro transcription in isolated cell nuclei. Acta Biotheoretica 44(1): 1-21.

Suarez, M. 2003. Scientific representation: Against similarity and isomorphism. International Studies in the Philosophy of Science 17(3): 225-44.

Wilke, H.-J. et al. 1994. A universal spine tester for in vitro experiments with muscle force simulation. European Spine Journal 3(2): 91-7.

Williams, J. A. (ed.) 2011. Predictive approaches in drug discovery and development: biomarkers and in vitro/in vivo correlations. Hoboken, N.J: Wiley.

Winsberg, E. 1999. Sanctioning models: The epistemology of simulation. Science in context 12(2): 275-92.

. 2003. Simulated experiments: Methodology for a virtual world. Philosophy of science 70(1): 105-25.

2009. A tale of two methods. Synthese 169(3): 575-92.

2014. Computer Simulations in Science. In: E. N. Zalta (ed.) The Stanford Encyclopedia of Philosophy. Fall 2014 edition.

Pío GARCíA

Universidad Nacional de Córdoba

Escuela de Filosofía

piogarcia@ffyh.unc.edu.ar

\section{Notas}

${ }^{1}$ La especificación de las condiciones metodológicas nos permitirá dar cuenta de la aparente circularidad de este esquema. En las consideraciones finales de este trabajo volveremos sobre este punto.

${ }^{2}$ Aquí se toma sólo uno de los sentidos destacados en la literatura que vincula representaciones con modelos (Cfr. Frigg \& Hartman 2012). Se podría retomar la discusión que sigue en términos de representación de modelos, pero, nos parece más adecuado seguir los términos en que ha sido planteado el problema en el contexto de la filosofía de la simulaciones

Principia 19(2): 217-234 (2015). 
computacionales. Aunque no será discutido en lo que sigue del trabajo, vale la pena señalar que habría otra forma de introducir el problema de la representaciones que estimamos podría ser más pertinente. Nos referimos a la propuesta de una perspectiva pragmática de la representación (Suarez 2003). Sin embargo, una discusión del alcance de propuestas como la de Suarez nos llevarían muy lejos de nuestro objetivo.

${ }^{3}$ En (Duran 2013) hay una discusión acerca de los alcances de este tipo de argumentación.

${ }^{4}$ A pesar de la perspectiva fuertemente ontológica que defiende Morgan, probablemente una de sus sugerencias más interesantes sea el señalamiento de que habría escenarios "híbridos" (experimentos materiales y no materiales) en las prácticas científicas contemporáneas (Morgan 2005).

${ }^{5}$ En los libros de textos se suele discriminar entre simulaciones basadas en agentes y aquellas basadas en modelos o ecuaciones (Cfr. Gilbert \& Troitzsch 2005).

${ }^{6}$ En otros trabajos hemos tomando otro sentido de exploración - uno cognitivo - para entender a las simulaciones (Garcia 2013; 2009).

${ }^{7}$ Estas ecuaciones deben ser modificadas en el sentido ya citado al principio de esta sección para que puedan ser consideradas como datos de entrada de un analizador diferencial (Cfr. Chance 1943, p.575).

${ }^{8}$ El proyecto entero detrás GEPASI ha sido reformulado recientemente por Pedro Mendes con un programa para la simulación de redes bioquímicas llamadas COPASI -complex pathway simulator. (Más información puede encontrarse en http://www.copasi.org)

${ }^{9}$ Los otros programas mencionados anteriormente se utilizan en contextos similares: Metamod es un paquete de software para estudiar las vías metabólicas de estado estacionario. Este paquete también permite el estudio de análisis de control de las rutas metabólicas. Scamp es un simulador de propósito general de reacciones metabólicas.

${ }^{10}$ En Garcia (2015) se desarrollan estos ejemplos de manera más extensa.

${ }^{11}$ El coeficiente de control flujo de una reacción es una de las nociones centrales del MCA.

${ }^{12}$ En Garcia (2006) hay una caracterización de tipo histórica de la relación entre condiciones in vivo-in vitro.

${ }^{13}$ Aquí podría citarse numerosos artículos en donde se utiliza el término 'simulación' para describir la relación entre condiciones in vivo e in vitro.

${ }^{14}$ Hartmann (1996) utiliza esta expresión aunque no desarrolla cuál sería su contenido específico.

Principia 19(2): 217-234 (2015). 